\title{
The recent X-ray history of NGC 5506
}

\author{
S. Bianchi ${ }^{1}$, I. Balestra ${ }^{1}$, G. Matt ${ }^{1}$, M. Guainazzi ${ }^{2}$, and G. C. Perola ${ }^{1}$ \\ ${ }^{1}$ Dipartimento di Fisica, Università degli Studi Roma Tre, Italy \\ 2 XMM-Newton Science Operation Center/RSSD-ESA, Villafranca del Castillo, Spain \\ Received 20 December 2002 / Accepted 11 February 2003
}

\begin{abstract}
We present a detailed discussion of the spectral and spatial components of NGC 5506, based on XMM-Newton, Chandra and BeppoSAX observations. The overall picture consists of a nucleus absorbed by cold gas with column density of $\approx 10^{22} \mathrm{~cm}^{-2}$ and surrounded by a Compton-thick torus, whose existence is inferred by a cold reflection component and an iron $\mathrm{K} \alpha$ line. On a much larger scale, a photoionized gas extended on $\approx 350 \mathrm{pc}$ reprocesses the nuclear radiation, producing a soft excess and ionized iron lines. Noteworthy, we find no evidence for the presence of the accretion disc.
\end{abstract}

Key words. galaxies: individual: NGC 5506 - galaxies: Seyfert - X-rays: galaxies

\section{Introduction}

NGC 5506 hosts a nearby $(z=0.006)$ AGN, rather bright in hard X-rays. It has been generally classified as a NELG. Recently Nagar et al. (2002) observed the source in the nearIR and discovered an heavily reddened $\left(A_{V} \sim 5\right)$ Narrow Line Seyfert 1 nucleus.

The source, being very bright, was observed by all X-ray satellites, starting with Uhuru. In recent times, it was observed by Einstein (Maccacaro et al. 1982), EXOSAT (Pounds et al. 1989) and Ginga (Bond et al. 1993); the latter fitted the spectrum with a power law absorbed by neutral matter with column density of $3-4 \times 10^{22} \mathrm{~cm}^{-2}$, plus a soft excess. Furthermore, the data showed a reflection component and an iron $\mathrm{K} \alpha$ line (Bond et al. 1993). Later on, ROSAT HRI images suggested that the soft emission is extended and coincident with the radio emission (Colbert 1998). Results from ASCA (Wang et al. 1999), BeppoSAX (Perola et al. 2002) and an RXTE variability analysis (Lamer et al. 2000) pointed out the complexity of the iron line profile, which was finally resolved by XMM-Newton in at least two components, the narrower at $6.4 \mathrm{keV}$ being likely associated with the reflection component, arising from a neutral Compton-thick material (Matt et al. 2001). The origin of the bluer and broader component is less clear, but may be associated to the soft excess (see Sect. 3.2.4). Finally, the source is variable on short time scales, but no long term trend has been found yet (e.g. Papadakis et al. 2002).

In this paper we present results from two XMM-Newton observations (the first one simultaneous with a BeppoSAX observation, see Matt et al. 2001) and a Chandra/HETG observation. We have also reanalysed past BeppoSAX and ASCA observations (see Table 1). All these observations will allow us

Send offprint requests to: $\mathrm{S}$. Bianchi, e-mail: bianchi@fis.uniroma3.it
Table 1. The log of all analysed observations, and exposure times.

\begin{tabular}{cccc}
\hline \hline Date & Mission & Instr. & $\boldsymbol{T}_{\text {exp }}(\mathbf{k s})$ \\
\hline $01 / 30 / 1997$ & ASCA & SIS0-1 & 39 \\
& & LECS & 16 \\
$01 / 30 / 1997$ & BeppoSAX & MECS & 39 \\
& & PDS & 17 \\
& & LECS & 9 \\
$01 / 14 / 1998$ & \multirow{2}{*}{ BeppoSAX } & MECS & 39 \\
& & PDS & 17 \\
$12 / 31 / 2000$ & \multirow{2}{*}{ Chandra } & ACIS-S HETG & 90 \\
& & LECS & 29 \\
$02 / 01 / 2001$ & \multirow{2}{*}{ BeppoSAX } & MECS & 78 \\
& & PDS & 38 \\
$02 / 02 / 2001$ & XMM-Newton & EPIC PN & 14 \\
$01 / 09 / 2002$ & XMM-Newton & EPIC PN & 10 \\
\hline & & &
\end{tabular}

to check and refine the interpretation proposed by Matt et al. (2001) and Bianchi \& Matt (2002), in which the nucleus is surrounded by at least two reflecting regions, one Compton-thick and neutral and the other one Compton-thin and highly ionized, and obscured by a Compton-thin cold absorber.

We will assume $H_{0}=75 \mathrm{~km} \mathrm{~s}^{-1} \mathrm{Mpc}^{-1}$ throughout the paper.

\section{Observations and data reduction}

\subsection{XMM-Newton}

NGC 5506 was observed twice by XMM-Newton, on February 2001 and on January 2002 (Table 1). We defer the reader to Matt et al. (2001) for details on the first observation and the related data reduction. Both observations were performed with the imaging CCD cameras, the EPIC-MOS 
(Turner et al. 2001) and the EPIC-pn (Strüder et al. 2001) operating in Large Window mode and the Medium filter. X-ray events corresponding to pattern 0 were used for the pn and 0-12 for the MOS. We will not deal with the RGS spectra in this paper, because of the too poor statistics. Data were reduced with SAS 5.3.0, including the first observation which was reprocessed. As an effect of the new adopted response matrix, we found a slightly different normalization factor between PDS and pn, 1.15, instead of 1.215 used by Matt et al. (2001). EPICpn spectra $(0.5-10 \mathrm{keV})$ and lightcurves were extracted from a radius of $40^{\prime \prime}$. An extraction region of $45^{\prime \prime}$ radii was instead adopted for the EPIC MOS1 and MOS2 spectra (0.3-10 keV). Both observations are affected by negligible pileup (less than $1 \%$ in the pn). Spectra were analysed with XSPEC 11.1.0.

\subsection{Chandra}

The Chandra observation was performed on New Year's Eve 2000 (Table 1) with the Advanced CCD Imaging Spectrometer (ACIS-S: Garmire et al., in preparation) and the High-Energy Transmission Grating Spectrometer (HETGS: Canizares et al., in preparation) in place. The high flux of the source together with the default frame time of $3.2 \mathrm{~s}$ resulted in a 0th order spectrum which is strongly affected by pileup (70\% according to WebPIMMS ${ }^{1}$ ). Therefore, we will only use the 1st order co-added MEG and HEG spectra in this paper. Data were reduced with the Chandra Interactive Analysis of Observations software (CIAO 2.2.1), using the Chandra Calibration Database (CALDB 2.10). Grating spectra were analysed with Sherpa 2.2.1.

\subsection{BeppoSAX}

BeppoSAX observed the source three times, on January 1997, January 1998 and February 2001 (Table 1). The first two observations were published by Risaliti (2002) and Perola et al. (2002), while the third one by Matt et al. (2001), together with the simultaneous XMM-Newton observation. Data reduction followed the standard procedure presented by Guainazzi et al. (1999), using HEAsoft 5.1. Spectra were extracted from regions of radius $8^{\prime}$ for the LECS and $4^{\prime}$ for the MECS, and analysed with XSPEC 11.1.0. A normalization factor of 0.86 was adopted between the PDS and the MECS (Fiore et al. 1999), appropriate for PDS spectra extracted with fixed rise time threshold.

\subsection{ASCA}

The source was observed by ASCA on January 1997, simultaneously with the first BeppoSAX observation. Data were published by Wang et al. (1999). SIS spectra $(0.5-10 \mathrm{keV})$ were downloaded from the ASCA Archive and background was extracted from blank field observations of SIS0 e SIS1. Data were analysed with HEAsoft 5.1. and XSPEC 11.1.0. Following the procedure adopted for the simultaneous XMM-BeppoSAX observation, we adopted a normalization factor of 0.898 between

\footnotetext{
${ }^{1}$ http://heasarc.gsfc.nasa.gov/Tools/w3pimms.html
}

the PDS and the SIS0 for the simultaneous ASCA-BeppoSAX observation.

In the following, errors are at the $90 \%$ confidence level for one interesting parameter $\left(\Delta \chi^{2}=2.71\right)$.

\section{Data analysis}

\subsection{Flux and spectral variability}

We analysed a total of seven X-ray observations of NGC 5506, covering a history of five years (see Table 1). The source shows a remarkable variability both during each observation (see Fig. 1a for an example), and between different observations (Fig. 1b). However, the large variation between the two XMM observations may not be related to a real longterm variability, because these observations are rather short. Therefore, the measured fluxes could be the effect of a large amplitude short-term variability, with the observations sampling different phases of it. This is also supported by the third long BeppoSAX observation, which has a average flux much higher than the first XMM-Newton one, which covers only a small fraction of it. Moreover, the three BeppoSAX observations have a remarkably similar average flux over a period of time of 4 years, suggesting that any variability time scale is smaller than about 1-2 days, i.e. the typical elapsed time of the BeppoSAX pointings.

The hardness ratio in each observation seems to indicate that there is no significant short-term spectral variation (Fig. 1a). On the other hand, in order to understand if the longterm flux variability is associated to spectral variations of the nuclear continuum, we performed a fit with a simple absorbed power law to all the analysed observations, with the only exception of Chandra, because the 0th order spectrum is largely affected by pileup (see Sect. 2.2), while in the 1st order spectra there are not enough continuum photons. We restricted our fits to the band $2.5-10 \mathrm{keV}$, in order to avoid the soft excess and the reflection component (we will deal with their temporal behaviour later). The results are shown in Figs. $2 \mathrm{a}$ and $\mathrm{b}$ : despite the large flux variability, no significant variations are present in the spectral shape of NGC 5506. Only the ASCA parameters (fully compatible with those derived by Wang et al. 1999) are systematically lower than the other ones but this is probably due to calibration problems, being inconsistent with the simultaneous BeppoSAX observation.

\subsection{Spectral analysis}

We start our spectral analysis from the three BeppoSAX observations, which give valuable informations on the broadband spectrum of the source. If we adopt a simple model with a power law absorbed by a column density of neutral gas, we get unacceptable fits (reduced $\chi^{2}$ higher than 2) for all the three datasets. Three features clearly arise from the residuals: a soft excess, an iron line and an excess at high energy, likely a signature of Compton reflection (see Fig. 3a for the third observation).

We note that the soft excess is an important component only in the LECS band and clearly does not affect the spectrum at 




(a)

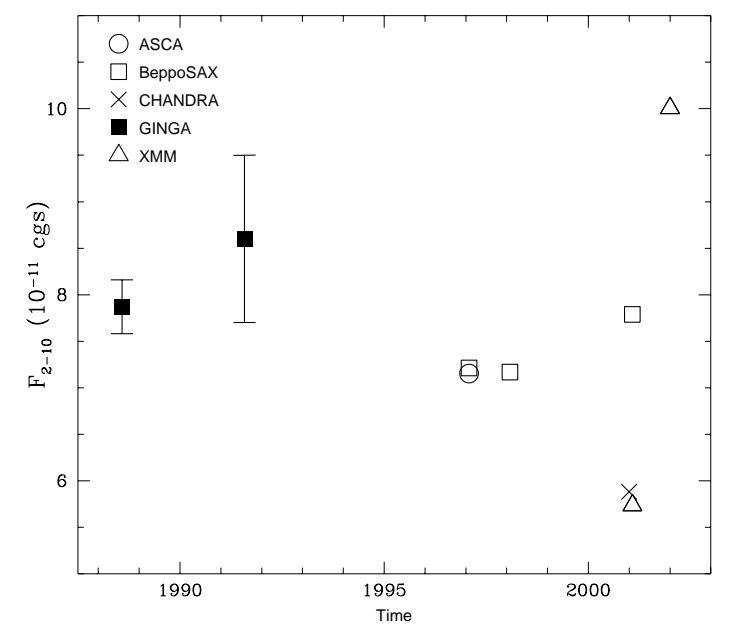

(b)

Fig. 1. a) Lightcurve and hardness ratio for the second XMM-Newton observation. b) Flux (2-10 keV) of NGC 5506 for all the observations analysed in this paper (see Table 1 for details). The two GINGA points were taken from Bond et al. (1993) and Nandra \& Pounds (1994).

higher energies. Therefore, we will exclude from our first analysis the LECS data and focus our attention in the next section on the reflection component and the iron line. The same reason led us to ignore all data below $2.5 \mathrm{keV}$ in the pn spectra, following Matt et al. (2001). The soft excess will be treated separately in Sect. 3.2.4 and put in the general context of the broadband model in Sect. 4.

\subsubsection{The broadband BeppoSAX data}

In order to correct the residuals on the high energy part of the spectrum, we changed our baseline model introducing a reflection component from neutral matter (PEXRAV model: Magdziarz $\&$ Zdziarski 1995) and an iron line. The same model successfully correct the residuals observed in the three BeppoSAX observations (see Fig. 3b).

The best fit energies of the iron lines are clearly shifted to energies higher than $6.4 \mathrm{keV}$ (respectively $6.69_{-0.13}^{+0.09}, 6.60_{-0.18}^{+0.07}$ and $6.57_{-0.07}^{+0.14} \mathrm{keV}$ for the three observations). Moreover,

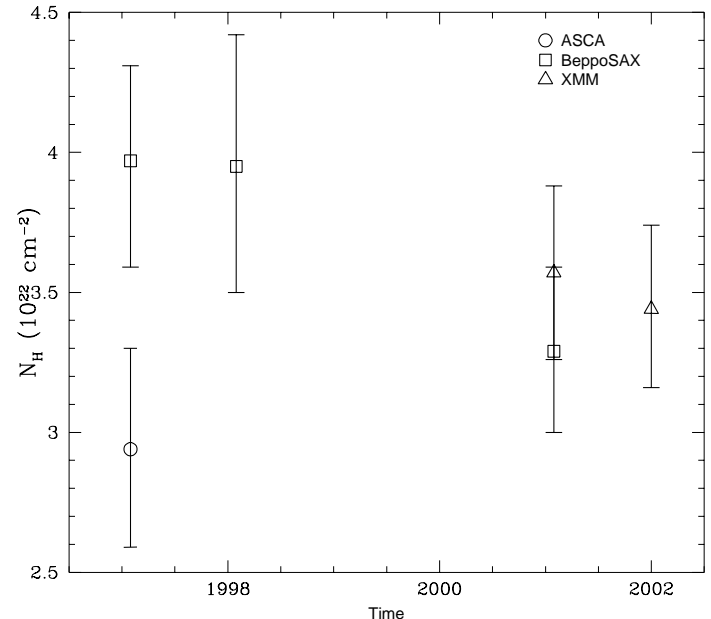

(a)

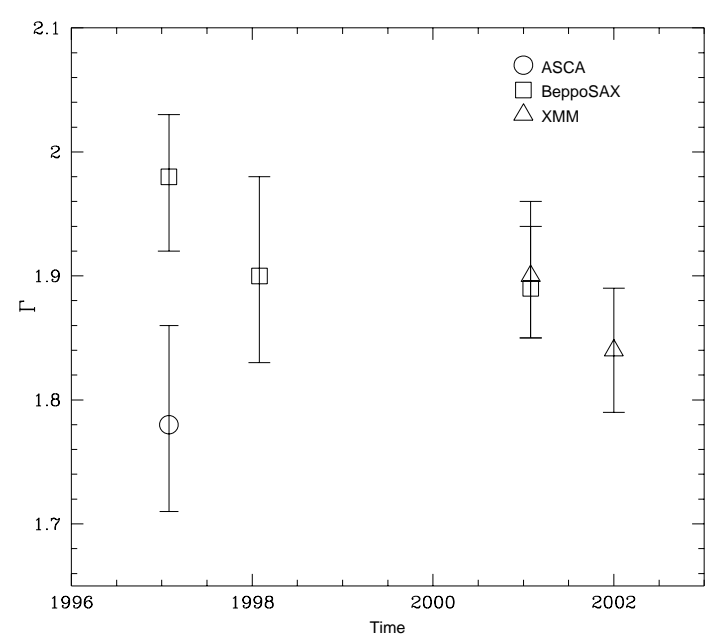

(b)

Fig. 2. Column density a) and photon index b) of NGC 5506 for the selected observations, adopting a simple absorbed power law model plus iron line in the $2.5-10 \mathrm{keV}$ band (see text for details).

the lines are resolved $(\simeq 0.3 \mathrm{keV})$. These results are consistent with those derived by Perola et al. (2002), but, interestingly, they are marginally different from the value of the line centroid in $A S C A$, being $6.44_{-0.04}^{+0.06}$, which in turns is consistent with the results published by Wang et al. (1999). The reason for this discrepancy is probably due to an intrinsic complexity of the iron line of this source combined with the different response of the two instruments at high energies.

Matt et al. (2001) found that the XMM-Newton data clearly show at least two components for the iron line, one narrow at $6.4 \mathrm{keV}$ and the other broad at higher energies. They suggested that the best explanation for the broad component is in terms of a blend of ionized iron lines, Fe Xxv at 6.7 and Fe XxvI at $6.96 \mathrm{keV}$. Therefore we decided to adopt a Compton reflection model plus three narrow Gaussian lines (with fixed centroid energies) also for the BeppoSAX data (see Table 2). All the other parameters do not change significantly from the initial model with a single, broad iron line, but the residuals around the line are better corrected and a marginal improvement of the $\chi^{2}$ is achieved. If this interpretation of the iron line complex 


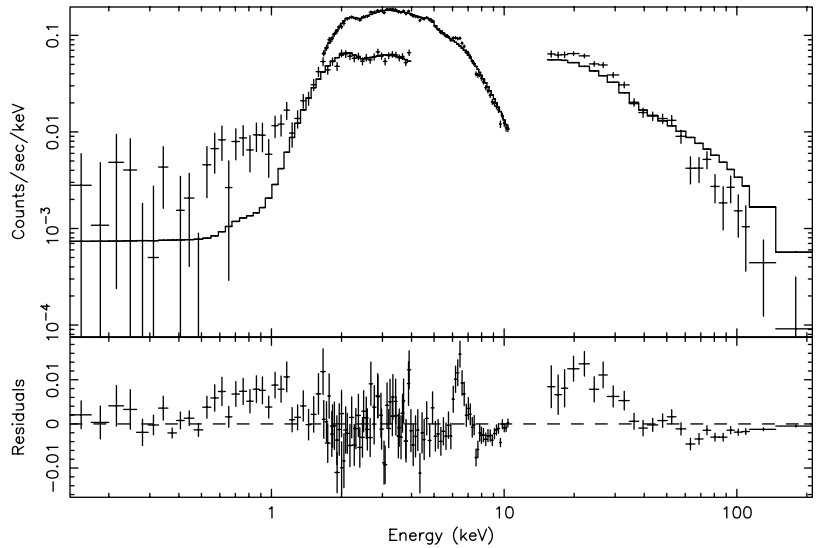

(a)

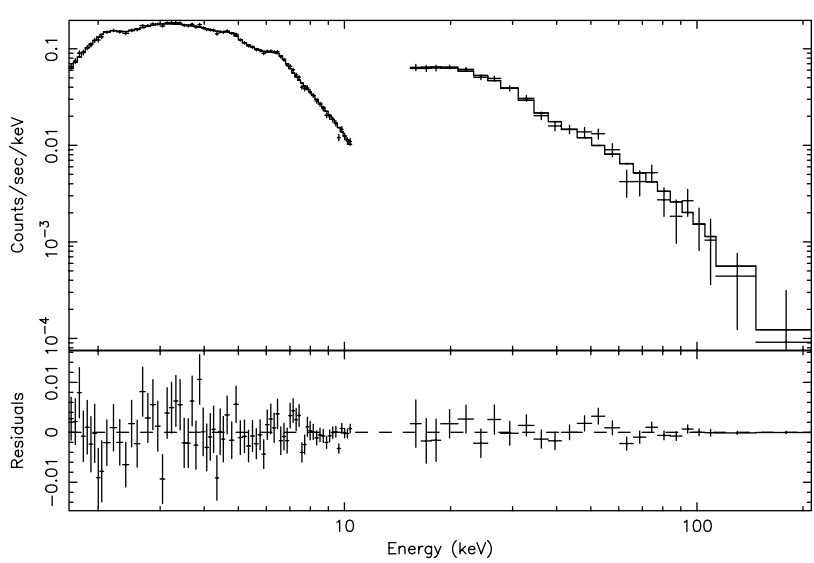

(b)

Fig. 3. a) Spectra and residuals from LECS, MECS and PDS from the last BeppoSAX observation when fitted with a simple absorbed power law. b) Spectra and residuals from MECS and PDS from the last BeppoSAX observation when a reflection component model plus iron line model is adopted (see text for details).

is correct, the ASCA observation of a single neutral iron line can be tentatively explained as due to the steeper decline of the effective area of this instrument at these energies.

The model with Compton reflection from neutral matter and three narrow Gaussian lines is statistically very good also for the pn-PDS spectrum from the simultaneous XMM-SAX observation (see third column in Table 3).

\subsubsection{The iron line complex}

As already mentioned, Matt et al. (2001) found that the pn spectrum showed an iron line composed by at least two components, one narrow, at $6.4 \mathrm{keV}$, and the other broader, bluewards. This structure for the line complex is less clear in the second XMM observations, where the total flux of the source was almost doubled and the broader component is less visible (see Fig. 4).

The line at $6.4 \mathrm{keV}$ is unresolved at the CCD resolution, in both the XMM observations. The higher spectral resolution of the gratings aboard Chandra allows a tighter upper limit on the FWHM of the line, which is about $4000 \mathrm{~km} \mathrm{~s}^{-1}(99 \%$ confidence level). This value clearly excludes an origin from
Table 2. Compton reflection plus three Gaussian lines model for the three BeppoSAX observations (MECS+PDS). The inclination angle of the reflecting matter is fixed to $30^{\circ}$ (see text for details).

\begin{tabular}{llll}
\hline \hline Date & $01 / 30 / 1997$ & $01 / 14 / 1998$ & $02 / 01 / 2001$ \\
\hline$\Gamma$ & $2.08_{-0.08}^{+0.07}$ & $1.93_{-0.10}^{+0.11}$ & $2.02_{-0.07}^{+0.08}$ \\
$N_{\mathrm{H}}\left(10^{22} \mathrm{~cm}^{-2}\right)$ & $3.77_{-0.19}^{+0.18}$ & $3.55_{-0.22}^{+0.21}$ & $3.42_{-0.14}^{+0.24}$ \\
$E_{\mathrm{c}}(\mathrm{keV})$ & $>318$ & $>169$ & $255_{-89}^{+235}$ \\
$R$ & $1.48_{-0.21}^{+0.44}$ & $1.02_{-0.38}^{+0.50}$ & $1.51_{-0.34}^{+0.42}$ \\
$F_{6.4 \mathrm{keV}}\left(10^{-5} \mathrm{ph} \mathrm{cm}^{-2} \mathrm{~s}^{-1}\right)$ & $7.9_{-1.8}^{+3.3}$ & $9.5_{-4.9}^{+4.9}$ & $8.6_{-5.0}^{+2.5}$ \\
$E W_{6.4 \mathrm{keV}}(\mathrm{eV})$ & $83_{-19}^{+35}$ & $94_{-48}^{+49}$ & $89_{-52}^{+26}$ \\
$E W_{6.7 \mathrm{keV}}(\mathrm{eV})$ & $<24$ & $<120$ & $<54$ \\
$E W_{6.96 \mathrm{keV}}(\mathrm{eV})$ & $68_{-42}^{+110}$ & $<72$ & $50_{-25}^{+70}$ \\
$F_{2-10 \mathrm{keV}}(\mathrm{cgs})$ & $7.2 \times 10^{-11}$ & $7.1 \times 10^{-11}$ & $7.8 \times 10^{-11}$ \\
$\chi^{2} /$ d.o.f. & $76 / 88$ & $92 / 82$ & $88 / 91$ \\
\hline
\end{tabular}

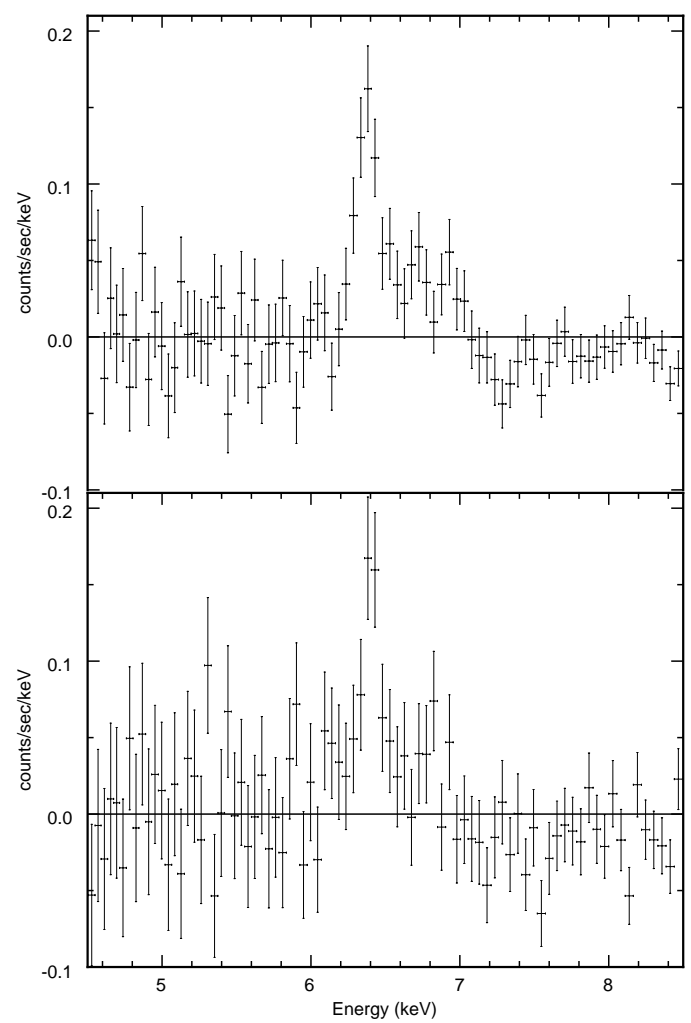

Fig. 4. EPIC-pn residuals around the iron line: first (top) and second (bottom) XMM observation.

the innermost region of an accretion disc, suggesting instead a more distant matter, such as the BLR or the torus. This is also supported by the absence of flux variability of the line in all the analysed observations (Fig. 6a). Because NGC 5506 was recently discovered to be a NLS1, with BLR line widths $<2000 \mathrm{~km} \mathrm{~s}^{-1}$ (Nagar et al. 2002), the upper limit to the line width from Chandra cannot permit to exclude an origin of the line from the BLR. On the other hand, it is preferable to identify the narrow line with the one inevitably arising from the matter producing the reflection component: we shall return on this subject in Sect. 4.1. It seems then reasonable that a torus is the most likely explanation for the $6.4 \mathrm{keV}$ line and the reflection at high energies. It is worth noting that this torus does not 


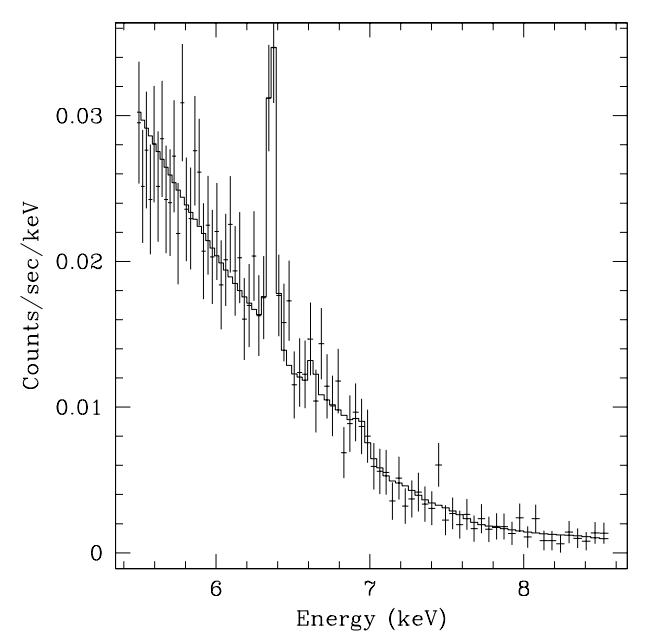

Fig. 5. The Chandra HEG spectrum of NGC 5506 around the iron line. The fit (solid line) includes three Gaussian lines at 6.4, 6.7 and $6.96 \mathrm{keV}$.

intercept the line of sight, as the column density of the absorber is too low to explain the observed line and Compton reflection continuum (see e.g. Matt et al. 2003). Therefore, in NGC 5506 both Compton-thin and Compton-thick circumnuclear matter are simultaneously present.

Following Matt et al. (2001) and the model we adopted for the BeppoSAX data, we interpreted the broad component of the iron line at higher energies as the blend of two ionized iron lines at 6.7 and $6.96 \mathrm{keV}$. Those lines have equivalent widths of a few tens eV in the first XMM observation (see Table 3) and around $10 \mathrm{eV}$ in the second one, when the flux of the continuum almost doubled. Indeed, even if they are often only upper limits, the fluxes of the two ionized lines are consistent to be constant among all the analysed observations (Figs. 6b, c). The ionized iron lines are not detected in the Chandra HEG spectrum (see Fig. 5). However, if two Gaussian lines are added to the fit at 6.7 and $6.96 \mathrm{keV}$, their upper limits are fully compatible with the flux measured in the XMM observations. The reason for the lack of detection in the grating spectra could be related to the physical origin of these lines and will be investigated in Sect. 3.2.4.

\subsubsection{An ionized disc?}

Even if the interpretation of the broad component of the iron line as a blend of two ionized lines successfully fits the data, we decided to test the alternative hypothesis that this component is actually the result of reprocessing by an ionized disc. This interpretation was tried and rejected by Matt et al. (2001), on the basis of a model including REFSCH and DISKLINE. We therefore decided to adopt a more refined model, xION (Nayakshin et al., in preparation), to fit the simultaneous pn/PDS spectrum. This model consists of reflection from a photoionized disc and the associated iron line, including relativistic effects. The adopted geometry is that of a lamp-post, with free parameters of the model being the height of the X-ray source above the disk, $h_{\mathrm{x}}$, the accretion rate through the disc, $\mathrm{m}$, the ratio between the luminosity of the X-ray source and that of the disc, $L_{\mathrm{X}} / L_{\mathrm{d}}$, the inner and outer disk radii (the first kept fixed to $3 R_{\mathrm{s}}$ in our fits) and the spectral index. We further added a narrow line at $6.4 \mathrm{keV}$ (which cannot be produced in an ionized disc) to this model. The fit is much worse than the one with reflection from neutral matter ( $\Delta \chi^{2}=31$ with 2 less d.o.f.), mostly due to a difficulty in reproducing the neutral iron edge at $7.1 \mathrm{keV}$. Therefore, it seems likely that most (if not all) the observed Compton reflection should be produced by a Compton-thick, cold material, where the neutral iron line is also likely produced. Thus, the following step was to add a further component, a Compton reflection from neutral matter modelled as PEXRAV with $R$ fixed to a negative value (to include only the reflected spectrum) and the spectral index linked to that of XION. The result is now statistically as good as the one obtained with only the Compton reflection and the three lines (see Table 3). Figure 7 shows the components of this model: most of the reflection at high energy, the iron edge and the narrow line are produced by neutral matter, while the broad component of the line originates from the ionized disc, whose reflected continuum does not contribute too much to the high energy spectrum.

However, the unbounded values of the distance of the illuminating lamp-post from the disc and the outer radius of the disc itself in the fit (see Table 3) are clearly indicating that there are not relativistic signatures on the spectrum. Moreover, the value of $L_{\mathrm{x}} / L_{\mathrm{d}}$ is very high, requiring an extreme fine-tuning for a magnetic flares model. These results are very similar to those obtained by Matt et al. (2001) with a model including PEXRAV, REFSCH and DISKLINE, which is just a less sophisticated physical picture of the one we adopted: the line is not very broad and requires a large outer radius for the disc (lack of relativistic signatures) and Compton reflection from the ionized disc is negligible if compared to that from neutral matter. These considerations make the ionized disc origin of the broad component less tenable than the identification as a blend of ionized lines, despite they are statistically equivalent.

\subsubsection{The soft excess}

The Chandra image of NGC 5506 clearly shows that the emission below $1 \mathrm{keV}$ is extended on a radius of $\simeq 350 \mathrm{pc}$ (Fig. 8). Furthermore, this region is clearly asymmetric with respect to the nuclear emission. Interestingly the dimension and the asymmetry of the X-ray emission seems to agree fairly well with the radio maps presented by Schmitt et al. (2001). On the other hand, no clear evidence of a possible extension on larger scale, as claimed by Colbert et al. (1998) on ROSAT HRI data, was found.

This extended gas is naturally associated to the soft excess best observed in the two XMM spectra and the ASCA one, allowing us to exclude thermal bremsstrahlung or a blackbody as possible explanations, since it is hard to imagine such a spectral shape arising from a $350 \mathrm{pc}$ scale gas. Therefore, in order to fit the residuals on the low energy part of the spectrum, we are left with two physically consistent models: reflection of the primary continuum from a photoionized gas or thermal emission from diffuse gas (MEKAL), which was found to give good fits in a sample of Compton-thin Seyfert 2s observed by BeppoSAX 


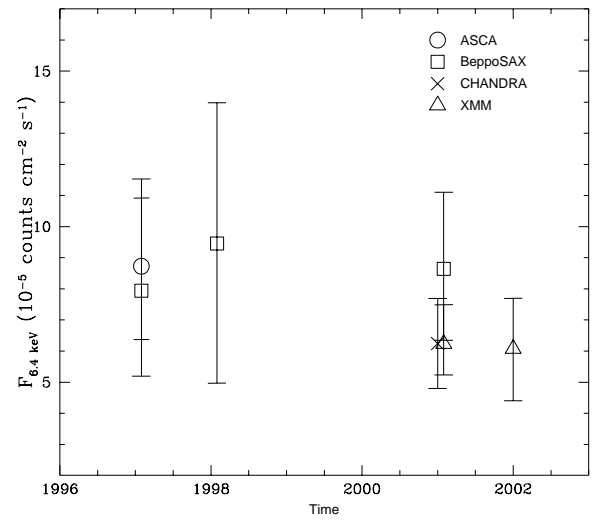

(a)

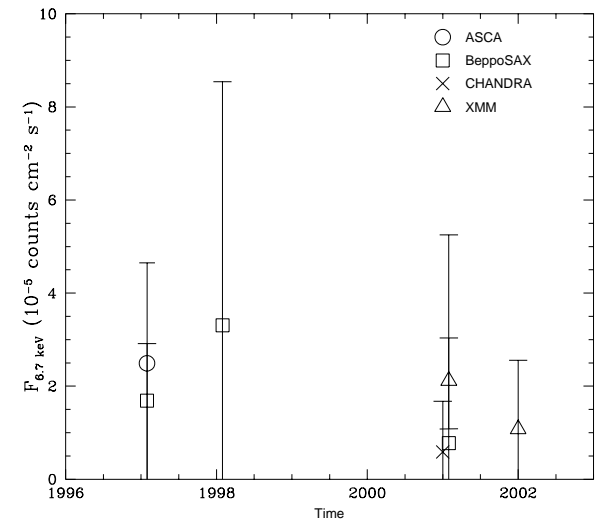

(b)

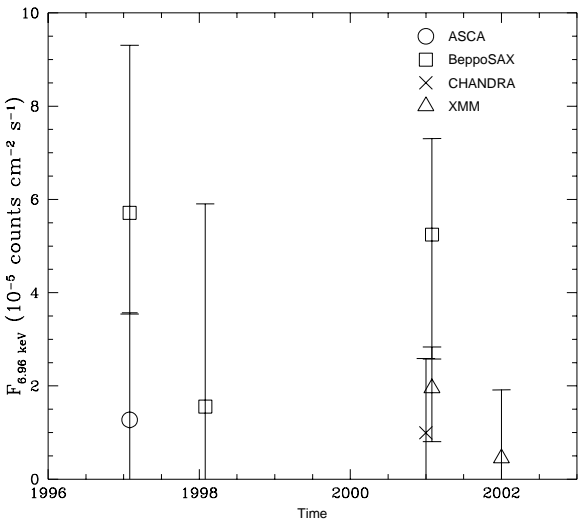

(c)

Fig. 6. Fluxes of the iron lines in the analysed observations of NGC 5506: 6.4 a), 6.7 b) and $6.96 \mathbf{c}$ ) keV. The centroid energies of the lines were kept fixed in the fits (see text for details).

Table 3. Best fit parameters for the simultaneous pn-PDS data above $2.5 \mathrm{keV}$. The two different adopted models are described in detail in Sects. 3.2.1 and 3.2.3.

\begin{tabular}{lll}
\hline \hline & XION+PEXRAV & PEXRAV+3 GAUSS \\
\hline$N_{\mathrm{H}}\left(10^{22} \mathrm{~cm}^{-2}\right)$ & $3.96_{-0.25}^{+0.29}$ & $3.53_{-0.28}^{+0.43}$ \\
$\Gamma$ & $2.05_{-0.07}^{+0.10}$ & $2.01_{-0.08}^{+0.10}$ \\
$E_{\mathrm{c}}(\mathrm{keV})$ & $1000_{-400}^{+1900}$ & $220_{-60}^{+490}$ \\
$R$ & - & $1.48_{-0.36}^{+0.54}$ \\
$h_{\mathrm{x}}\left(R_{\mathrm{s}}\right)$ & $>39$ & - \\
$L_{\mathrm{x}} / L_{\mathrm{d}}$ & $20.0_{-1.5}^{+1.9}$ & - \\
$\dot{m}($ Eddington rate $)$ & $<0.85$ & - \\
Outer radius $\left(R_{\mathrm{s}}\right)$ & $>150$ & - \\
$E W_{6.4 \mathrm{keV}}(\mathrm{eV})$ & $68_{-15}^{+9}$ & $88_{-13}^{+20}$ \\
$E W_{6.7 \mathrm{keV}}(\mathrm{eV})$ & - & $27_{-12}^{+17}$ \\
$E W_{6.96 \mathrm{keV}}(\mathrm{eV})$ & - & $41_{-27}^{+11}$ \\
$\chi^{2} /$ d.o.f. & $152 / 170$ & $150 / 172$ \\
\hline
\end{tabular}

(Risaliti 2002). The first one is parametrized (when self absorption effects are neglected) by a power law with the spectral index linked to that of the primary PEXRAV continuum responsible for the photoionization of the gas itself. We further allowed for absorption by an intervening column density of neutral gas. The best fits for the two models are presented in Table 4: even if marginally better from a statistical point of view, the MEKAL fits have metal abundances unphysically low. This means that the resulting spectrum lacks emission lines and is virtually indistinguishable from the already rejected bremsstrahlung interpretation. Reflection from photoionized gas remains then the only tenable model. The observed intervening absorption is $\simeq 2 \times 10^{21} \mathrm{~cm}^{-2}$, in excess of the Galactic one, and is possibly due to the host galaxy, which is seen almost edge-on.

As expected from an origin from diffuse gas, the flux of this component is remarkably constant between the two XMM observations (see Table 4), while the flux of the primary component almost doubles (see Sect. 2). Therefore, the ratio of the soft excess to the nuclear flux varies from 2 to $1 \%$ in the two observations. If the interpretation as reflection from photoionized

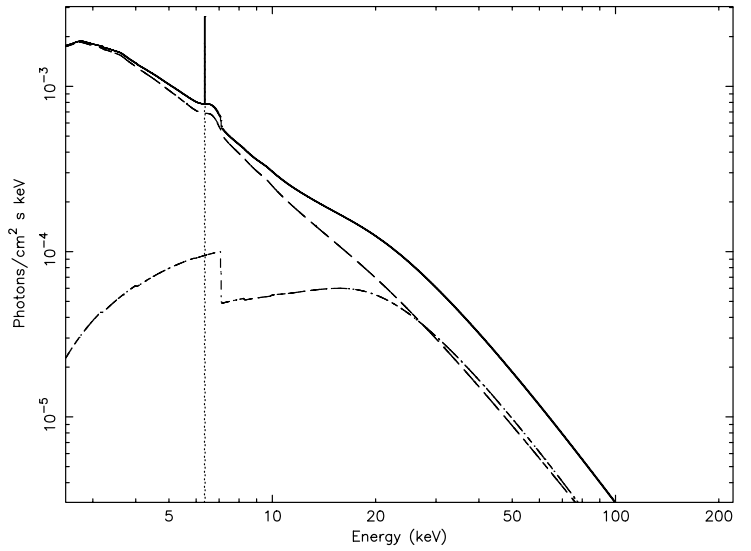

Fig. 7. A model including a cut-off power law reprocessed by an ionized disc and Compton-thick neutral matter, plus a narrow line at $6.4 \mathrm{keV}$ (see text for details).

matter is correct, then the column density of this gas should be a few times $10^{22} \mathrm{~cm}^{-2}$. Bianchi \& Matt (2002) have calculate the equivalent widths of the ionized iron lines produced in gas photoionized by a power law continuum. They found that equivalent widths (with respect to the total continuum, primary plus reflected) of the order of tens of $\mathrm{eV}$ can indeed be produced by a photoionized gas with a column density around $10^{22} \mathrm{~cm}^{-2}$ and a modest iron overabundance.

In order to understand if our model of reflection from photoionized matter is still tenable when the gas is distributed on the observed scale, we performed a test with cLoudy. The density of this gas was assumed to have a $r^{-2}$ behaviour, normalized in order to produce the inferred column density of $\simeq 10^{22} \mathrm{~cm}^{-2}$ at a radius of $\simeq 350 \mathrm{pc}$. The incident continuum was taken with the $2-10 \mathrm{keV}$ observed mean luminosity and spectral shape. We have found a solution with an ionization structure fully compatible with the production of ionized iron lines (see Bianchi \& Matt 2002, for details), which does not require a temperature higher than $10^{5} \mathrm{~K}$. Such temperatures make thermal emission from the gas completely negligible, so the model is self-consistent. Moreover, if the gas where they are emitted is spatially extended, the dispersion by the gratings would 


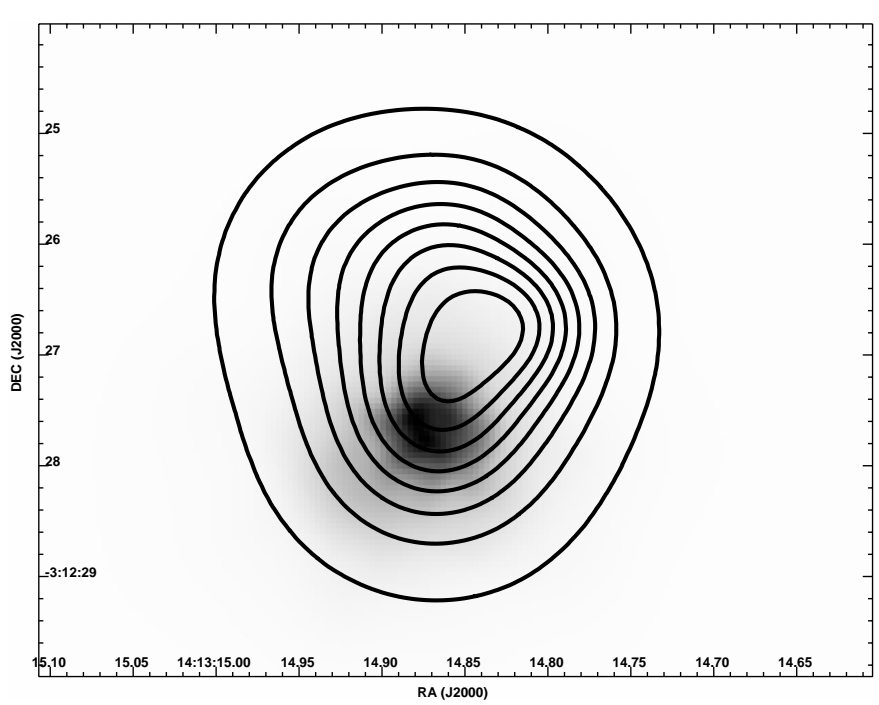

Fig. 8. Chandra contour levels of the emission below $1 \mathrm{keV}$ superimposed over the total emission.

become rather complicated making the ionized lines difficult to detect: this would be a reason for the lack of their detection in the Chandra HEG spectrum (see Sect. 3.2.2). Unfortunately, the statistic is too poor to directly search for extended line emission in the Chandra images.

\section{Discussion}

In this section we summarize the results on all the components of the X-ray spectrum of NGC 5506 and put them in the context of a general scenario.

\subsection{The broadband model}

The central engine is a Seyfert 1 nucleus, whose spectrum is as usual represented by a power law with an exponential cutoff. The nuclear emission is reflected from a Compton-thick neutral material, producing the characteristic "bump" at high energies (e.g. Matt et al. 1991). The narrow $6.4 \mathrm{keV}$ iron line and the $7.1 \mathrm{keV}$ edge from neutral iron are likely to originate from the same matter. The value of the equivalent width of the line is fully compatible with the amount of the Compton reflection. However, a possible iron underabundance is suggested by the data (see Table 5): an $E W$ of about $135 \mathrm{eV}$ would be expected if $R=0.75, \theta=30^{\circ}$ and $\Gamma=1.9$ (George \& Fabian 1991; Matt et al. 1991). In particular, the line, being narrow and keeping a constant flux despite large variation in the total flux of the source, constrains this material to be fairly distant from the black hole. This makes a parsec scale torus the best identification for this gas. It is interesting to note that the only evidence for the presence of a Compton-thick torus in NGC 5506 is indirect, being the signatures of reflection of the nuclear radiation on its inner walls.

The direct nuclear emission and the reflection components are obscured by another neutral material, with a column density of $N_{\mathrm{Hc}} \simeq 3 \times 10^{22} \mathrm{~cm}^{-2}$. The presence of Compton-thick and Compton-thin matter in the circumnuclear regions of the same
Table 4. Best fit parameters for the models adopted to explain the soft excess in the two XMM observations (pn+MOS1+MOS2) and the ASCA one. The fluxes are relative to the power law model.

\begin{tabular}{llll}
\hline \hline & ASCA97 & XMM01 & XMM02 \\
\hline POWERLAW & & & \\
$\chi^{2} /$ d.o.f. & $702 / 702$ & $674 / 611$ & $727 / 611$ \\
$N_{\mathrm{Hs}}\left(10^{21} \mathrm{~cm}^{-2}\right)$ & $1.8_{-0.7}^{+0.3}$ & $1.5_{-0.1}^{+0.2}$ & $1.7_{-0.2}^{+0.2}$ \\
& & & \\
\hline MEKAL & & & \\
$\chi^{2} /$ d.o.f. & $689 / 700$ & $665 / 609$ & $717 / 609$ \\
$N_{\mathrm{Hs}}\left(10^{21} \mathrm{~cm}^{-2}\right)$ & $2.8_{-0.8}^{+2.3}$ & $1.9_{-0.3}^{+0.3}$ & $2.1_{-0.5}^{+0.5}$ \\
$k T(\mathrm{keV})$ & $0.73_{-0.17}^{+0.14}$ & $0.80_{-0.13}^{+0.20}$ & $0.68_{-0.12}^{+0.15}$ \\
$A_{Z}$ & $0.03_{-0.02}^{+0.07}$ & $<0.02$ & $<0.02$ \\
& & & \\
\hline Flux 0.5-1 keV & & & \\
$\left(10^{-13} \mathrm{cgs}\right)$ & $1.80 \pm 0.01$ & $2.14 \pm 0.01$ & $2.22 \pm 0.01$ \\
\hline
\end{tabular}

source seems to be a widespread phenomenon in the environment of Seyfert galaxy (see e.g. Matt et al. 2003, and references therein). In this respect, NGC 5506 is yet another example.

Extended on a region of $350 \mathrm{pc}$ radii, a gas, photoionized by the nuclear radiation, surrounds the nucleus. The primary continuum is simply reflected, producing a component which is again a power law with the same photon index as the incident spectrum. The total column density of this gas is $\simeq 10^{22} \mathrm{~cm}^{-2}$, calculated from the ratio between the normalizations of the reflected and the nuclear continuum. The ionization structure of this gas is such that ionized iron lines at 6.7 and $6.96 \mathrm{keV}$ are produced, with equivalent widths against the total continuum of tens of $\mathrm{eV}$. The radiation reflected from this gas is further obscured by a neutral gas along the line of sight with column density of $N_{\mathrm{Hs}} \simeq 2 \times 10^{21} \mathrm{~cm}^{-2}$, in excess to that of our Galaxy. This gas could naturally be associated with the host galaxy, which is seen almost edge-on.

The best fit parameters for this model are shown in Table 5, when used on the pn-MOS-PDS and the SIS-PDS spectra. The results for the two sets of simultaneous observations are consistent with each other, with the only exception of the photon index and the column density $N_{\mathrm{Hc}}$. The marginal inconsistency of these values could be partly due to the fact that the MOS spectra were fitted down to $0.3 \mathrm{keV}$, while the SIS ones to $0.5 \mathrm{keV}$, introducing some discrepancies in the determination of the spectral index and the cold absorption.

\subsection{Where is the accretion disc?}

It is worth noting that our model does not contain any component directly related to the accretion disc. Indeed, the results from the fits with the model XION (see Sect. 3.2.3) require quite extreme conditions and basically confirm that there is no signature of reprocessing from the accretion disc in our data. There are at least two possible explanations to this problem.

It is possible that the accretion disc is completely ionized. In this case, the reflection component would be almost indistinguishable from the primary continuum, being a simple power law. Moreover, neither iron lines nor edges would be produced 
Table 5. Best fit parameters for the broadband model adopted for NGC 5506 on the pn-MOS-PDS and the SIS-PDS spectra (see text for details).

\begin{tabular}{lcc}
\hline \hline & pn-MOS-PDS (2001) & SIS-PDS (1997) \\
\hline$\Gamma$ & $1.81_{-0.06}^{+0.03}$ & $1.94_{-0.08}^{+0.10}$ \\
$E_{\mathrm{c}}(\mathrm{keV})$ & $130_{-40}^{+35}$ & $150_{-50}^{+140}$ \\
$R$ & $0.75_{-0.11}^{+0.15}$ & $1.4_{-0.5}^{+0.8}$ \\
$N_{\mathrm{Hc}}\left(10^{22} \mathrm{~cm}^{-2}\right)$ & $2.89_{-0.04}^{+0.02}$ & $3.2_{-0.1}^{+0.1}$ \\
$N_{\mathrm{Hs}}\left(10^{21} \mathrm{~cm}^{-2}\right)$ & $1.52_{-0.19}^{+0.13}$ & $1.9_{-0.8}^{+0.8}$ \\
$E W_{6.4 \mathrm{keV}}(\mathrm{eV})$ & $86_{-10}^{+22}$ & $88_{-32}^{+24}$ \\
$E W_{6.7 \mathrm{keV}}(\mathrm{eV})$ & $26_{-9}^{+9}$ & $30_{-24}^{+19}$ \\
$E W_{6.96 \mathrm{keV}}(\mathrm{eV})$ & $28_{-11}^{+10}$ & $<24$ \\
$F_{2-10 \mathrm{keV}}(\mathrm{cgs})$ & $5.8 \times 10^{-11}$ & $6.9 \times 10^{-11}$ \\
$\chi^{2} /$ d.o.f. & $709 / 627$ & $441 / 438$ \\
\hline
\end{tabular}

because all the elements would be completely stripped off, leaving no signatures for the presence of the disc, at least in the energy band the cold absorption present in NGC 5506 let us investigate (i.e. above a few keV). Very ionized accretion discs are generally produced by high accretion rates (e.g. Ballantyne et al. 2001, and references therein). It is possible to estimate the accretion rate for NGC 5506 from the mass of the central BH, as inferred from Hayashida et al. (1998) from X-ray variability. Even if their value is highly model dependent, a mass of $2 \times 10^{6} M_{\odot}$ would produce an Eddington luminosity of $3 \times 10^{44} \mathrm{erg} \mathrm{s}^{-1}$. Starting from the mean unabsorbed $2-10 \mathrm{keV} X$-ray luminosity we observed $\left(\approx 6 \times 10^{42} \mathrm{erg} \mathrm{s}^{-1}\right)$, we can derive the bolometric luminosity of the source by multiplying by a factor 10-30 (Elvis et al. 1994). Therefore, the Eddington ratio for NGC 5506 is $\eta \approx 0.2-0.6$, which in turns means a high accretion rate. Low mass $\mathrm{BHs}$ and high values of the Eddington ratio are generally believed to be typical characteristics of NLS1s, as physical explanations for the spectral peculiarities of these sources (see e.g. Boller et al. 1996; Laor et al. 1997; Boroson 2002). In this respect, ionized discs could be ubiquitous in NLS1s as a result of the required high accretion rate and recently there have been claims of their presence in these sources (see Ballantyne et al. 2001, and references therein).

A second solution would be that the accretion disc is seen almost edge-on, making the reprocessed components hardly detectable because of their low intensity (see e.g. Puchnarewicz et al. 1991). This solution, even if not implausible, is not favoured by the fact that the torus clearly does not intercept the line of sight. Therefore, a geometry would be required with the disc and the torus which are not co-aligned as would be, at least naively, expected. Interestingly, such a scenario is required in another NLS1, RE J1034+396, whose inferred values of BH mass and Eddington ratio are of the same order as NGC 5506: this source is best modelled by an almost edge-on disc, while no torus again intercepts the line of sight (Puchnarewicz et al. 2001).

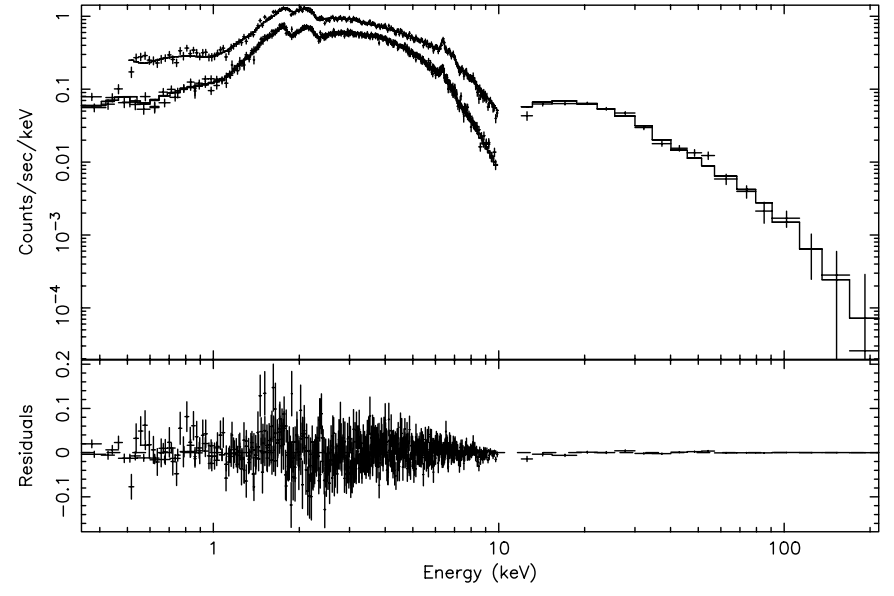

Fig. 9. Best fit model for the pn-MOS-PDS data (see text and Table 5 for details).

\section{Conclusions}

The analysis of all the data available from XMM-Newton, Chandra, BeppoSAX and ASCA led us to picture a selfconsistent scenario for the X-ray emission of the NLS1 NGC 5506:

- Both Compton-thin and Compton-thick circumnuclear matter are simultaneously present: the former intercepts the line of sight and absorbs the source flux at soft energies; the latter is indirectly observed through the signatures left in the spectrum, a Compton reflection component, a neutral iron edge and a narrow $\mathrm{K} \alpha$ line.

- The iron line consists of a narrow neutral component, arising from the torus, and a blend of ionized iron lines at 6.7 and $6.96 \mathrm{keV}$.

- The nucleus is surrounded by an extended $(\approx 350 \mathrm{pc})$ soft emission, likely associated with reflection of the nuclear emission by a photoionized gas, where both the soft excess and the ionized iron lines are produced.

- There is no spectral signature for the presence of the accretion disc: possible solutions could be a fully ionized or almost edge-on disc.

Acknowledgements. SB, IB, GM and GCP acknowledge ASI and MIUR (under grant cofIN-00-02-36) for financial support. We would like to thank Sergei Nayakshin for his helpful comments on the XION code, and the anonymous referee.

\section{References}

Ballantyne, D. R., Iwasawa, K., \& Fabian, A. C. 2001, MNRAS, 323, 506

Bianchi, S. \& Matt, G. 2002, A\&A, 387, 76

Boller, T., Brandt, W. N., \& Fink, H. 1996, A\&A, 305, 53

Bond, I. A., Matsuoka, M., \& Yamauchi, M. 1993, ApJ, 405, 179

Boroson, T. A. 2002, ApJ, 565, 78

Colbert, E. J. M., Baum, S. A., O’Dea, C. P., \& Veilleux, S. 1998, ApJ, 496, 786

Elvis, M., Wilkes, B. J., McDowell, J. C., et al. 1994, ApJS, 95, 1 
Fiore, F., Guainazzi, M., \& Grandi, P. 1999, SDC report (http://www.asdc.asi.it/bepposax/software/ index.html)

George, I. M., \& Fabian, A. C. 1991, MNRAS, 249, 352

Guainazzi, M., Perola, G. C., Matt, G., et al. 1999, A\&A, 346, 407

Hayashida, K., Miyamoto, S., Kitamoto, S., Negoro, H., \& Inoue, H. 1998, ApJ, 500, 642

Lamer, G., Uttley, P., \& McHardy, I. M. 2000, MNRAS, 319, 949

Laor, A., Fiore, F., Elvis, M., Wilkes, B. J., \& McDowell, J. C. 1997, ApJ, 477, 93

Maccacaro, T., Perola, G. C., \& Elvis, M. 1982, ApJ, 257, 47

Magdziarz, P. \& Zdziarski, A. A. 1995, MNRAS, 273, 837

Matt, G., Perola, G. C., \& Piro, L. 1991, A\&A, 247, 25

Matt, G., Guainazzi, M., Perola, G. C., et al. 2001, A\&A, 377, L31

Matt, G., Guainazzi, M., \& Maiolino, R. 2003, MNRAS, in press [astro-ph/0302328]

Nagar, N. M., Oliva, E., Marconi, A., \& Maiolino, R. 2002, A\&A, 391, L21
Nandra, K., \& Pounds, K. A. 1994, MNRAS, 268, 405

Nayakshin, S., Kallman, T. R., \& Kazanas, D., in preparation, http://lheawww.gsfc.nasa.gov/users/serg/ms.ps

Papadakis, I. E., Petrucci, P. O., Maraschi, L., et al. 2002, ApJ, 573, 92

Perola, G. C., Matt, G., Cappi, M., et al. 2002, A\&A, 389, 802

Pounds, K. A., Nandra, K., Stewart, G. C., \& Leighly, K. 1989, MNRAS, 240, 769

Puchnarewicz, E. M., Mason, K. O., Siemiginowska, A., et al. 2001, ApJ, 550, 644

Risaliti, G. 2002, A\&A, 386, 379

Schmitt, H. R., Ulvestad, J. S., Antonucci, R. R. J., \& Kinney, A. L. 2001, ApJS, 132, 199

Strüder, L., Briel, U., Dennerl, K., et al. 2001, A\&A, 365, L18

Turner, M. J. L., Abbey, A., Arnaud, M., et al. 2001, A\&A, 365, L27

Wang, T., Mihara, T., Otani, C., Matsuoka, M., \& Awaki, H. 1999, ApJ, 515, 567 